\title{
AUTHOR INDEX Volume 12 (2014)
}

Afra, S., see Gao, S.

2 (2014) 1450025

Akhmedov, M., see Karabulut, N. P.

2 (2014) 1441007

Akhtar, M. N., Southey, B. R., Andrén, P. E., Sweedler, J. V. and Rodriguez-Zas, S. L., Identification of best indicators of peptide-spectrum match using a permutation resampling approach

5 (2014) 1440001

Akulenko, R., see Chen, J.

5 (2014) 1442005

5 (2014) 1441008

5 (2014) 1450025

Alhajj, R., see Gao, S.

5 (2014) 1450007

Alhajj, R., see Qabaja, A.

5 (2014) 1450027

Al-Turaiki, I., see Badr, G.

5 (2014) 1440001

Andrén, P. E., see Akhtar, M. N.

5 (2014) 1441006

Badr, G., Al-Turaiki, I., Turcotte, M. and Mathkour, H., IncMD: Incremental trie-based structural motif discovery algorithm

5 (2014) 1450027

Bahi, J. M., see Guyeux, C.

Bayar, B., Bouaynaya, N. and Shterenberg, R., Probabilistic

5 (2014) 1350017 non-negative matrix factorization: Theory and application to microarray data analysis

$1(2014) 1450001$

Bednarz, P. and Wilczyński, B., Supervised learning method for predicting chromatin boundary associated insulator elements

6 (2014) 1442006

Bienia, W., see Guyeux, C.

6 (2014) 1350017

Borštnik, B. and Pumpernik, D., The apparent enhancement of $\mathrm{CpG}$ transversions in primate lineage is a consequence of multiple replacements

3 (2014) 1450011

Bouaynaya, N., see Bayar, B.

3 (2014) 1450001

Chakrabarty, B. and Parekh, N., PRIGSA: Protein repeat identification by graph spectral analysis

6 (2014) 1442009 
Chen, J., Lutsik, P., Akulenko, R., Walter, J. and Helms, V., AKSmooth: Enhancing low-coverage bisulfite sequencing data via kernel-based smoothing

6 (2014) 1442005

Chiang, J.-H. and Ju, J.-H., Discovering novel protein-protein interactions by measuring the protein semantic similarity from the biomedical literature

6 (2014) 1442008

Cho, Y.-R., see Jaromerska, S.

Choi, M.-H., Kang, S.-H. and Lim, H.-S., An improved 6 (2014) 1450004 preprocessing algorithm for haplotype inference by pure parsimony

$4(2014) 1450020$

Choudhari, S., Lohia, R. and Grigoriev, A., Comparative metagenome analysis of an Alaskan glacier

2 (2014) 1441003

Chua, H. N., see Vazifedoost, A.

Cokol, M., see Karabulut, N. P.

2 (2014) 1450014

2 (2014) 1441007

Côté, N. M.-L., see Guyeux, C.

2 (2014) 1350017

Dantan, E., see Sanchez-Osorio, I.

2 (2014) 1330003

Datta, A., see Inamdar, H.

2 (2014) 1450026

De Rose, C. A. F., see Dias, R.

2 (2014) 1450013

Demetrick, D., see Gao, S.

2 (2014) 1450025

2 (2014) 1450021

Dias, R., Xavier, M. G., Rossi, F. D., Neves, M. V., Lange, T. A. P., Giongo, A., De Rose, C. A. F. and Triplett, E. W., MPI-blastn and NCBI-TaxCollector: Improving metagenomic analysis with high performance classification and wide taxonomic attachment

3 (2014) 1450013

Dias, U., Galvão, G. R., Lintzmayer, C. N. and Dias, Z., A general heuristic for genome rearrangement problems

3 (2014) 1450012

Dias, Z., see Dias, U.

Duan, J., Zhang, J.-G., Wan, M., Deng, H.-W. and Wang, Y.-P., Population clustering based on copy number variations detected from next generation sequencing data

Eisenhaber, F. and Sherman, W. A., 10 years for the Journal 3 (2014) 1450012 of Bioinformatics and Computational Biology (2003-2013) - A retrospective

Eisenhaber, F., Unix interfaces, Kleisli, bucandin structure, etc. — The heroic beginning of bioinformatics in Singapore

Ellis, J., see Ren, J. 3 (2014) 1450023

Elsheikh, A., see Qabaja, A. 3 (2014) 1450007

Eskin, E., see He, D. 3 (2014) 1442007

Eslahchi, C., see Rezvan, A. 3 (2014) 1450028

Exarchos, K. P., see Lampros, C.

3 (2014) 1450016 
Exarchos, T. P., see Lampros, C.

3 (2014) 1450016

Fang, C., Noguchi, T. and Yamana, H., Analysis of evolutionary conservation patterns and their influence on identifying protein functional sites

5 (2014) 1440003

Fesenko, E. E., see Purtov, Y. A.

5 (2014) 1441006

Fotiadis, D. I., see Lampros, C.

5 (2014) 1450016

5 (2014) 1350014

Gadkari, R. A., see Rakshambikai, R.

5 (2014) 1450012

Gao, S., Karakira, I., Afra, S., Naji, G., Alhajj, R., Zeng, J. and Demetrick, D., Evaluating predictive performance of network biomarkers with network structures

5 (2014) 1450025

Gennemark, P. and Wedelin, D., ODEion - A software module for structural identification of ordinary differential equations

1 (2014) 1350015

1 (2014) 1450010

Ghomi, H. T., Thompson, J. J. and Lill, M. A., Are distancedependent statistical potentials considering three interacting bodies superior to two-body statistical potentials for protein structure prediction?

5 (2014) 1450022

Ghosh, K. and Schlipf, J., Formal modeling of a system of chemical reactions under uncertainty

5 (2014) 1440002

Gill, J., Jayaswal, P. and Salunke, D. M., Antigen exposure leads to rigidification of germline antibody combining site

Giongo, A., see Dias, R.

Glazunova, O. A., see Purtov, Y. A.

Goebels, F., see Krivozubov, M.

Goto, S., see Kotera, M.

Grigoriev, A., see Choudhari, S.

Guo, F.-B., see Ru, B.

Guyeux, C., Côté, N. M.-L., Bahi, J. M. and Bienia, W., Is protein folding problem really a NP-complete one?

First investigations

3 (2014) 1450006

3 (2014) 1450013

3 (2014) 1441006

3 (2014) 1441004

3 (2014) 1442001

3 (2014) 1441003

3 (2014) 1450005

1 (2014) 1350017

Hammond, D. A., Olman, V. and Xu, Y., Functional understanding of the diverse exon-intron structures of human GPCR genes

1 (2014) 1350019

He, D. and Eskin, E., IPED2X: A robust pedigree reconstruction algorithm for complicated pedigrees

6 (2014) 1442007

Helms, V., see Chen, J.

6 (2014) 1442005

Hernaez, M., see Ochoa, I.

6 (2014) 1442002

Holloway, D. M., see Zagrijchuk, E. A.

6 (2014) 1441009

Huang, J., see Ru, B.

6 (2014) 1450005

Hüllermeier, E., see Mernberger, M.

6 (2014) 1350016 
Inamdar, H., Datta, A., Manjari K, S. and Joshi, R., Rule-based integration of RNA-Seq analyses tools for identification of novel transcripts

5 (2014) 1450026

Irausquin, S., see Mukhopadhyay, R.

5 (2014) 1450002

Ito, S., see Ueno, Y.

5 (2014) 1442003

Jarada, T., see Qabaja, A.

5 (2014) 1450007

Jaromerska, S., Praus, P. and Cho, Y.-R., Distance-wise pathway discovery from protein-protein interaction networks weighted by semantic similarity

1 (2014) 1450004

Jayaswal, P., see Gill, J.

1 (2014) 1450006

Jin, G., see Xia, F.

1 (2014) 1450008

Joshi, R., see Inamdar, H.

1 (2014) 1450026

Ju, J.-H., see Chiang, J.-H.

1 (2014) 1442008

Kanehisa, M., see Kotera, M.

1 (2014) 1442001

1 (2014) 1450020

Karabulut, N. P., Akhmedov, M. and Cokol, M., A drug similarity network for understanding drug mechanism of action

$2(2014) 1441007$

Karacaören, B., Admixture mapping of growth related traits in $\mathrm{F}_{2}$ mice dataset using ancestry informative markers

Karakira, I., see Gao, S.

Katayama, T., see Kotera, M.

Kawashima, S., see Kotera, M.

Khlebodarova, T. M., see Likhoshvai, V. A.

Kim, S., see Koo, I.

King, R. D. and Lu, C., An investigation into eukaryotic pseudouridine synthases

2 (2014) 1441010

2 (2014) 1450025

2 (2014) 1442001

2 (2014) 1442001

2 (2014) 1450009

2 (2014) 1450018

$4(2014) 1450015$

Kobiki, S. and Maruyama, O., ReSAPP: Predicting overlapping protein complexes by merging multiplesampled partitions of proteins

6 (2014) 1442004

6 (2014) 1442003

Konagaya, A., see Ueno, Y.

Koo, I., Yao, S., Zhang, X. and Kim, S., Comparative analysis of false discovery rate methods in constructing metabolic association networks

Kordyukova, L. V., see Mintaev, R. R.

4 (2014) 1450018

4 (2014) 1441008

Kotera, M., Nishimura, Y., Nakagawa, Z.-I., Muto, A., Moriya, Y., Okamoto, S., Kawashima, S., Katayama, T., Tokimatsu, T., Kanehisa, M. and Goto, S., PIERO ontology for analysis of biochemical transformations: Effective implementation of reaction information in the IUBMB enzyme list 6 (2014) 1442001

Kozlov, K. N., see Myasnikova, E.

6 (2014) 1441002 
Krivozubov, M., Goebels, F. and Spirin, S., Estimation of relative effectiveness of phylogenetic programs by machine learning

Lampros, C., Simos, T., Exarchos, T. P., Exarchos, K. P., Papaloukas, C. and Fotiadis, D. I., Assessment of optimized Markov models in protein fold classification

Lange, T. A. P., see Dias, R.

Li, J., see Ren, J.

Li, M., see Wooley, J.

Likhoshvai, V. A. and Khlebodarova, T. M., Mathematical modeling of bacterial cell cycle: The problem of coordinating genome replication with cell growth

Lill, M. A., see Ghomi, H. T.

Lim, H.-S., see Choi, M.-H.

Lin, H., see Ru, B.

Lindhardt, E. and Gennemark, P., Automated analysis of routinely generated preclinical pharmacokinetic and pharmacodynamic data

Lintzmayer, C. N., see Dias, U.

Lohia, R., see Choudhari, S.

Lu, C., see King, R. D.

Lukyanov, V. I., see Purtov, Y. A.

Lutsik, P., see Chen, J.

Maier, U. G., see Mernberger, M.

Makarenkov, V., see Willems, M.

Manjari K, S., see Inamdar, H.

Marashi, S.-A., see Rezvan, A.

Maruyama, O., see Kobiki, S.

Mathkour, H., see Badr, G.

Mayorga, P., see Sanchez-Osorio, I.

Mernberger, M., Moog, D., Stork, S., Zauner, S., Maier, U. G. and Hüllermeier, E., Protein sub-cellular localization prediction for special compartments via optimized time series distances

Miller, J., see Taillefer, E.

Mintaev, R. R., Alexeevski, A. V. and Kordyukova, L. V., Co-evolution analysis to predict protein-protein interactions within influenza virus envelope

Mizuta, S., see Shida, F.

Moog, D., see Mernberger, M.

Moriya, Y., see Kotera, M.

Moshiri, B., see Vazifedoost, A.
2 (2014) 1441004

4 (2014) 1450016

4 (2014) 1450013

4 (2014) 1450023

4 (2014) 1401001

3 (2014) 1450009

3 (2014) 1450022

3 (2014) 1450020

3 (2014) 1450005

3 (2014) 1450010

3 (2014) 1450012

3 (2014) 1441003

3 (2014) 1450015

3 (2014) 1441006

3 (2014) 1442005

3 (2014) 1350016

3 (2014) 1450024

3 (2014) 1450026

3 (2014) 1450028

3 (2014) 1442004

3 (2014) 1450027

3 (2014) 1330003

1 (2014) 1350016

1 (2014) 1350018

2 (2014) 1441008

2 (2014) 1450019

2 (2014) 1350016

2 (2014) 1442001

2 (2014) 1450014 
Mukhopadhyay, R., Irausquin, S., Schmidt, C. and Valafar, H., DynaFold: A dynamic programming approach to protein backbone structure determination from minimal sets of residual dipolar couplings

1 (2014) 1450002

Muto, A., see Kotera, M.

Myasnikova, E. and Kozlov, K. N., Statistical method for

1 (2014) 1442001 estimation of the predictive power of a gene circuit model

2 (2014) 1441002

Naji, G., see Gao, S.

Nakagawa, Z.-I., see Kotera, M.

2 (2014) 1450025

Neves, M. V., see Dias, R.

2 (2014) 1442001

2 (2014) 1450013

Ng, S. K., see Vazifedoost, A.

2 (2014) 1450014

$\mathrm{Ni}, \mathrm{Q}$. and Zou, L., Accurate discrimination of outer membrane proteins using secondary structure element alignment and support vector machine

1 (2014) 1450003

Nie, F., see Ru, B.

Nishimura, Y., see Kotera, M.

Noguchi, T., see Fang, C.

1 (2014) 1450005

1 (2014) 1442001

1 (2014) 1440003

Ochoa, I., Hernaez, M. and Weissman, T., Aligned genomic data compression via improved modeling

6 (2014) 1442002

6 (2014) 1442001

Okamoto, S., see Kotera, M.

6 (2014) 1350019

Olman, V., see Hammond, D. A.

6 (2014) 1441006

6 (2014) 1450016

Papaloukas, C., see Lampros, C.

6 (2014) 1442009

6 (2014) 1450017

6 (2014) 1441006

6 (2014) 1450004

6 (2014) 1441006

6 (2014) 1450011

Pumpernik, D., see Borštnik, B.

Purtov, Y. A., Glazunova, O. A., Antipov, S. S., Pokusaeva, V. O., Fesenko, E. E., Preobrazhenskaya, E. V., Shavkunov, K. S., Tutukina, M. N., Lukyanov, V. I. and Ozoline, O. N., Promoter islands as a platform for interaction with nucleoid proteins and transcription factors Qabaja, A., Jarada, T., Elsheikh, A. and Alhajj, R.,

Prediction of gene-based drug indications using compendia of public gene expression data and PubMed abstracts

2 (2014) 1441006

Rahgozar, M., see Vazifedoost, A.

3 (2014) 1450007

3 (2014) 1450014

Rakshambikai, R., Srinivasan, N. and Gadkari, R. A.,

Repertoire of protein kinases encoded in the genome of zebrafish shows remarkably large population of PIM kinases 
Ramos, F., see Sanchez-Osorio, I.

1 (2014) 1330003

Ren, J., Ellis, J. and Li, J., Influenza A HA's conserved epitopes and broadly neutralizing antibodies: A prediction method

5 (2014) 1450023

Rezvan, A., Marashi, S.-A. and Eslahchi, C., FCDECOMP:

Decomposition of metabolic networks based on flux coupling relations

5 (2014) 1450028

5 (2014) 1440001

Rodriguez-Zas, S. L., see Akhtar, M. N.

Rogacheva, O. N., Stefanov, V. E., Shchegolev, B. F., Vershinina, E. A. and Savvateeva-Popova, E. V., Role of arginine 209 in the conformational transition of the protein kinase A regulatory subunit $\mathrm{RI} \alpha$ A-domain

Rossi, F. D., see Dias, R.

Ru, B., 't Hoen, P. A. C., Nie, F., Lin, H., Guo, F.-B. and Huang, J., PhD7Faster: Predicting clones propagating faster from the Ph.D.-7 phage display peptide library

Sabirov, M. A., see Zagrijchuk, E. A.

1 (2014) 1450005

1 (2014) 1441009

Sadeghi, M., see Vazifedoost, A.

Salunke, D. M., see Gill, J.

1 (2014) 1450014

1 (2014) 1450006

Sanchez-Osorio, I., Ramos, F., Mayorga, P. and Dantan, E., Foundations for modeling the dynamics of gene regulatory networks: A multilevel-perspective review

2 (2014) 1441005

2 (2014) 1450013

Savvateeva-Popova, E. V., see Rogacheva, O. N.

Schlipf, J., see Ghosh, K.

Schmidt, C., see Mukhopadhyay, R.

Shavkunov, K. S., see Purtov, Y. A.

Shchegolev, B. F., see Rogacheva, O. N.

Sherman, D. J., see Zhukova, A.

Sherman, W. A., see Eisenhaber, F.

Shida, F. and Mizuta, S., Measurement of word frequencies in genomic DNA sequences based on partial alignment and fuzzy set

4 (2014) 1450019

Shterenberg, R., see Bayar, B.

4 (2014) 1450001

Simos, T., see Lampros, C.

Southey, B. R., see Akhtar, M. N.

4 (2014) 1450016

Spirin, S., see Krivozubov, M.

4 (2014) 1440001

4 (2014) 1441004

Spirov, A. V., see Zagrijchuk, E. A.

4 (2014) 1441009

Srinivasan, N., see Rakshambikai, R.

4 (2014) 1350014

Stefanov, V. E., see Rogacheva, O. N.

4 (2014) 1441005

Stork, S., see Mernberger, M.

4 (2014) 1350016 
Suratanee, A. and Plaimas, K., Identification of inflammatory bowel disease-related proteins using a reverse $k$-nearest neighbor search

4 (2014) 1450017

Sweedler, J. V., see Akhtar, M. N.

't Hoen, P. A. C., see Ru, B.

Tahiri, N., see Willems, M.

4 (2014) 1440001

4 (2014) 1450005

4 (2014) 1450024

Taillefer, E. and Miller, J., Exhaustive computation of exact duplications via super and non-nested local maximal repeats

1 (2014) 1350018

1 (2014) 1450022

Thompson, J. J., see Ghomi, H. T.

Tokimatsu, T., see Kotera, M.

1 (2014) 1442001

Triplett, E. W., see Dias, R.

1 (2014) 1450013

Turcotte, M., see Badr, G.

Tutukina, M. N., see Purtov, Y. A.

1 (2014) 1450027

1 (2014) 1441006

Ueno, Y., Ito, S. and Konagaya, A., Implementing a modeling software for animated protein-complex interactions using a physics simulation library

6 (2014) 1442003

Valafar, H., see Mukhopadhyay, R.

Vazifedoost, A., Rahgozar, M., Moshiri, B., Sadeghi, M., Chua, H. N., Ng, S. K. and Wong, L., Using data fusion for scoring reliability of protein-protein interactions

6 (2014) 1450002

Vershinina, E. A., see Rogacheva, O. N.

Walter, J., see Chen, J.

Wan, M., see Duan, J.

Wang, Y.-P., see Duan, J.

Wedelin, D., see Gennemark, P.

Weissman, T., see Ochoa, I.

Wilczyński, B., see Bednarz, P.

Willems, M., Tahiri, N. and Makarenkov, V., A new efficient algorithm for inferring explicit hybridization networks following the Neighbor-Joining principle

Wong, L., see Wooley, J.

Wong, L., see Vazifedoost, A.

Wooley, J., Li, M. and Wong, L., JBCB, the first decade

Xavier, M. G., see Dias, R.

Xia, F. and Jin, G., Fine-grained parallelism accelerating for RNA secondary structure prediction with pseudoknots based on FPGA

3 (2014) 1450008

$\mathrm{Xu}, \mathrm{Y}$., see Hammond, D. A.

3 (2014) 1350019

Yamana, H., see Fang, C.

3 (2014) 1440003

Yao, S., see Koo, I.

3 (2014) 1450018 
Zagrijchuk, E. A., Sabirov, M. A., Holloway, D. M. and Spirov, A. V., In silico evolution of the hunchback gene indicates redundancy in cis-regulatory organization and spatial gene expression

Zauner, S., see Mernberger, M.

Zeng, J., see Gao, S.

Zhang, J.-G., see Duan, J.

Zhang, X., see Koo, I.

Zhukova, A. and Sherman, D. J., Knowledge-based generalization of metabolic networks: A practical study Zou, L., see Ni, Q.
2 (2014) 1441009

2 (2014) 1350016

2 (2014) 1450025

2 (2014) 1450021

2 (2014) 1450018

2 (2014) 1441001

2 (2014) 1450003 